Published in final edited form as:

J Empir Res Hum Res Ethics. 2012 April ; 7(2): 3-19. doi:10.1525/jer.2012.7.2.3.

\title{
Research Ethics Education for Community-Engaged Research: A Review and Research Agenda
}

\author{
Emily E. Anderson, \\ Loyola University Chicago
}

Stephanie Solomon,

Saint Louis University

Elizabeth Heitman,

Vanderbilt University

James M. DuBois,

Saint Louis University

Celia B. Fisher,

Fordham University

Rhonda G. Kost,

The Rockefeller University

Mary Ellen Lawless,

Case Western Reserve University

Cornelia Ramsey,

Virginia Commonwealth University

Bonnie Jones,

University of North Carolina

Alice Ammerman, and

University of North Carolina

Lainie Friedman Ross

University of Chicago

\section{Abstract}

Community engagement is increasingly becoming an integral part of research. "Communityengaged research" (CEnR) introduces new stakeholders as well as unique challenges to the protection of participants and the integrity of the research process. We-a group of representatives of CTSA-funded institutions and others who share expertise in research ethics and CEnR-have identified gaps in the literature regarding (1) ethical issues unique to CEnR; (2) the particular instructional needs of academic investigators, community research partners, and IRB members; and (3) best practices for teaching research ethics. This paper presents what we know, as well as what we still need to learn, in order to develop quality research ethics educational materials tailored to the full range of stakeholder groups in CEnR. 


\section{Keywords}

community-based participatory research; community-engaged research; research ethics education

"Community engagement" refers broadly to what the Carnegie Foundation for the Advancement of Teaching describes as "collaboration between institutions of higher education and their larger communities (local, regional/state, national, global) for the mutually beneficial exchange of knowledge and resources in a context of partnership and reciprocity" (http://classifications.carnegiefoundation.org/descriptions/ community_engagement.php?key=1213).

Community engagement is a growing area of interest in many health-related disciplines; it is also integrated into an increasing number of federally funded research programs, including the National Institutes of Health (NIH) Clinical and Translational Science Awards (CTSA) (Hood et al., 2010) and other extramural research grants from NIH and the Centers for Disease Control and Prevention (CDC) (for example, see http://www.cdc.gov/prc/). NIH has adopted the broad term "community-engaged research" (CEnR) to describe research that involves collaboration among stakeholders whose common goal is to improve health, regardless of the specific types or degrees of engagement (Clinical and Translational Science Awards Community Engagement Key Function Committee Task Force on the Principles of Community Engagement, 2011). We apply this term to "research that provides communities with a voice and role in the research process beyond providing access to research participants," and consider engagement to include studying the views of community members regarding research protocols, community advisory and review boards, hiring community members as part of a research team, and including community members as co-investigators (DuBois et al., 2011).

We are a group of representatives of CTSA-funded institutions and others who share expertise in research ethics and CEnR (see Table 1, which delineates the numerous roles that the authors play in CEnR ethics, research ethics education, and research ethics curriculum development). We convened via multiple teleconferences over the course of about one year. Our initially identified goal was to identify, develop, and disseminate research ethics educational programs and materials that could meet the unique needs of communityacademic research partnerships.

As a first step, we attempted to reach consensus regarding learning objectives, content, and approaches for research ethics instruction, tailored instructional materials, and quality measures. Our discussions have highlighted the fact that there is limited evidence regarding: (1) the particular instructional needs of academic investigators, community research partners, and IRB members; (2) best practices for teaching research ethics; and (3) effective ways to integrate CEnR's unique ethical issues into research ethics education. Based on our collective experiences, review of relevant literatures, and extensive deliberation, this manuscript presents what is known, as well as what still needs to be learned, in order to develop quality research ethics educational materials that are tailored to the full range of stakeholder groups in CEnR. In order to provide background and context for our recommendations regarding best practices and a research agenda, we will first outline unique ethical issues that arise in CEnR; describe the various stakeholder groups that require ethics education specific to CEnR; and present current standard methods of delivering research ethics education and discuss their limitations for community research partners. 


\section{Unique Ethical Issues that Arise in CEnR}

There are numerous ethical issues that are unique to CEnR or that manifest uniquely in the CEnR context. Many of these have not been fully explored from all stakeholders' perspectives; gaps in knowledge and lack of solid guidance makes integration of these issues into research ethics education difficult. Here we categorize these ethical challenges as a first step in determining topics of salience for research ethics education for all stakeholder groups.

\section{Partnership Challenges}

Many ethical problems that arise in CEnR are not directly related to participant protections or scientific integrity; rather, they stem from the fact that academic and community partners sometimes have overlapping but incongruent goals, disparate power and access to resources, or different work and communication styles (Lindau et al., 2011; Shore, 2006; Silka \& Renault-Caragianes, 2006). For example, community organizations may feel overburdened by academic partners' requests for input on formal products such as grant proposals or manuscripts, particularly if they have a limited number of paid staff members. Partner organizations may be eager to deliver services to community members and feel stifled by the sometimes slow pace of research and intensive attention to process. Successful collaboration requires a strong mutual commitment to power-sharing and open communication about the collaborative process, inherent assumptions, and stakeholder goals (Heitman \& McKieran, 2004).

\section{Community vs. Academic Expertise}

Community and academic researchers have different kinds of expertise. Although respecting partners' expertise is a central tenet of ethical engagement (Fisher \& Goodman, 2009; Fisher \& Ragsdale, 2006), negotiating decisions when experts conflict can be challenging. Threats to the protection of participants or the integrity of the research process may arise from the fact that community partners view their communities from a unique vantage point; they have knowledge about their members that academic researchers cannot learn simply from studying the community, and articulating these community dynamics to outsiders may be difficult. For example, community partners working as recruiters in community-based drug trials have observed that study inclusion/exclusion criteria sometimes do not reflect the realities of participants' lives (Fisher et al., 2012; True, Alexander, \& Fisher, 2012).

However, they may not feel empowered to communicate their concerns to investigators. Life experiences may also attune community partners to certain things about which universitybased investigators may be naïve, such as when a participant may be lying in order to enroll in a study. Problems may arise if community partners do not feel comfortable scrutinizing potential participants in order to verify eligibility or to make screening or eligibility decisions beyond strictly adhering to written protocol instructions.

There has long been a perceived tension between "good science" and community engagement (Buchanan, Miller, \& Wallerstein, 1997). Particular protocol features, such as the collection of a large amount of personal information, may create suspicion about researchers' intentions (Alexander \& Richman, 2008). Community partners may also question the fairness of certain research methodologies such as the use of a control group (Fisher \& Wallace, 2000). If academic researchers do not adequately explain the reasons for certain methodological decisions, community partners may question their integrity or even their scientific expertise. 


\section{Risks to Groups}

Traditional clinical research ethics frameworks focus almost exclusively on risks to individuals. Ross et al. (2010b) have highlighted risks to individuals qua community (group) membership (if the individual self-identifies as a group member or is externally identified) and risks that occur to the group qua group as key considerations in CEnR. Harms to individuals can occur during the research process when a person is labeled and recruited because of membership in a group. For example, in a culture where blood carries special meaning, if blood samples are taken for a research study, individual participants may be at risk for stigmatization within their community. Harms to individuals can also result from research outcomes if findings about a group are attributed to an individual member. For example, because research has shown that BRCA1 and BRCA2 genes are more prevalent in persons of Ashkenazi Jewish heritage, increased risk for breast cancer is attributed to any woman who describes herself as Ashkenazi Jewish (whether or not she participated in the research), potentially resulting in increased insurance premiums (Weijer, 1999). CEnR can also pose risks to individual agency as a result of group membership. For example, if the leader of a disease group has had a negative research experience, he or she may refuse to provide a researcher with access to the group for a project that may be of significant benefit to the group, leaving others in the group entirely unaware of the opportunity (Ross et al., 2010b).

Risks to communities also include potential disruptions to group structure and function. For example, internal conflict resulting from disagreement over aspects of a community's research participation can lead to diminished group cohesiveness. The following example demonstrates potential disruption to both internal and external relationships as well as group agency. The Havasupai Native American tribe was concerned about growing rates of diabetes in their community and gave blood samples to investigators whom they had asked to conduct genetic research on diabetes. Ultimately, the investigators concluded that novel genetic factors were not a primary factor in the Havasupai's diabetes problem. These same blood samples were also used to study genetic factors in other diseases, including schizophrenia, and may have also been shared with researchers at other institutions. The samples were also used to determine that the tribe originated in Asia and migrated across the Bering Strait to what is now Arizona. This finding significantly threatens the tribe's traditional collective belief that they originated from the Grand Canyon. However, neither tribe leaders nor the individuals who provided the samples gave consent or even knew about these additional uses of the blood samples (Ross et al., 2010b).

\section{Threats to Informed Consent, Voluntariness, and the Integrity of Data}

Community partners' specific roles and general stature within their communities can influence participants' perceptions of a study and their decisions to enroll (and continue participating) (Gikonyo et al., 2008). It is often argued that community involvement leads to increased trust, higher participant enrollment, and perhaps even improved informed consent (Minkler, 2004; Quinn, 2004; Strauss et al., 2001). However, much as academic investigators may be misperceived as bringing services to communities of research interest, community research partners may be mistakenly viewed as service providers, that is, individuals who "know best" and have participants' best interests at heart and resources to offer (Terpstra et al., 2011). Bean and Silva (2010) make a comparison between peer recruiters and clinician-researchers, whose preexisting, trusting relationships with potential participants create potential conflicts of interest in recruitment (Fisher, 2012; Levine, 1992).

Even if an individual community partner does not hold a formal leadership position in a community, close proximity and likeness to potential research participants may create subtle peer pressure to participate. It has been suggested that peer-driven recruitment shares some 
of the characteristics of network marketing (e.g., similar to home-based sales parties to sell jewelry, cosmetics, or housewares). When a potential participant is approached by a recruiter who is also a peer, the participant assumes the recruiter is playing the role of peer first and recruiter second (Phillips, 2010). This perception mediates potential participants' concerns about risks and benefits and ultimately their willingness to participate in a research study.

Community partners may struggle to balance the expectations of their community members with the requirements of a research study and expectations of their academic partners (Brugge \& Cole, 2003; Terpstra et al., 2011). There is increasing pressure from federal funders to meet recruitment goals, and much CEnR is conducted in vulnerable communities where participation in research has been historically low. Pressure to meet recruitment goals (and perhaps a perception that enrolling participants is more important than providing complete information and ensuring understanding and voluntariness) can lead to protocol violations, de-emphasis on risks, pressuring friends and family to participate (Terpstra et al., 2011), or falsifying data (True, Alexander, \& Richman, 2011).

\section{Expectations of Benefits}

Despite being presented with similar information, people associate a variety of different benefits with research participation (e.g., development of programs and services, direct health-related feedback). Recent research suggests that perceptions about benefits may vary among racial/ethnic groups (Lakes et al., 2011). To complicate matters, CEnR projects often do include both research and nonresearch activities (e.g., direct service provision, community health education). This may lead community partners to conflate research and service, not because they do not conceptually understand the difference, but because their primary goal is to obtain services for their communities, and they believe the programs offered to be beneficial (or at least better than nothing) (True, Alexander, \& Richman, 2011). A desire to provide presumed benefits to community members may lead partners to target certain subgroups or kinds of people, such as those individuals whom they think are deserving or who will be more compliant, thus creating selection bias (Terpstra et al., 2011).

\section{Potential Conflicts of Interest}

Community partners may be employed by or otherwise affiliated with community organizations that are involved in research partnerships, or they may be hired by an academic institution explicitly to liaise with the community for research purposes. No matter their role in or specific contributions to the research, their primary employers, their priorities, and their allegiances may vary (Alexander \& Richman, 2008; Terpstra et al., 2011). For example, community partners may interact within their community as service providers or caregivers in addition to being engaged in research. They may be health care providers, public health professionals, teachers, community organizers, medical assistants, or social workers; these dual roles may present competing personal or professional obligations (Fisher et al., 2012; True, Alexander, \& Fisher, 2012). They may also be fathers, grandmothers, writers, or housepainters, and their nonresearch roles may value social and ethical norms that differ from research norms, creating ethical ambiguity or conflicts of interest (Anderson, 2010). They may have other interests that could impact the ways in which participants are recruited. For example, members of a patient advocacy group may have personal interests in seeing a study reach enrollment goals if they or family members share the medical condition being studied, and this could lead to an emphasis on benefits and de-emphasis on risks during the informed consent process (Landy \& Sharp, 2010). 


\section{Moral Distress}

Research suggests that community partners can experience moral distress, defined in the nursing literature as "the inability of a moral agent to act according to his or her core values and perceived obligations due to internal and external constraints" (Ulrich, Hamric, \& Grady, 2010), when conducting research in communities in which they live and/or work. Moral distress can arise when community partners learn certain information about their communities (e.g., high rates of disease and disability) and become concerned about the circumstances of research participants with whom they interact, particularly if they are members of the same community (Simon \& Mosavel, 2010). They may feel a sense of responsibility for things that are beyond their research role- at potential peril to themselves and participants. Community partners may be frustrated by their inability to provide immediate assistance to members of their communities due to limited resources and/or the fact that the primary goal of research is not service provision (Fisher et al., 2012; True, Alexander, \& Fisher, 2012). A propensity to want to help can compromise study findings (e.g., randomization) and informed consent (Terpstra et al., 2011). Moral distress may also lead to feelings of disempowerment, particularly when community partners disagree with recruitment approaches, inclusion criteria, or other aspects of study design.

\section{Control over Interpretation and Dissemination of Research Results}

When it comes time to interpret and disseminate findings, academic and community partners may have competing agendas. Academic investigators may focus on generalizable claims in order to secure publication or additional funding, while community groups may focus on the local relevance of findings and want to use data for securing services or changing public policy. Partners may have conflicting ideas regarding how and where data are presented as well as what data should be published (Ross et al., 2010a).

\section{Who Needs Research Ethics Education?}

Efforts to provide appropriate education and skills training in research ethics are typically motivated by two separate but related goals. The first is an essential role of academic institutions: to ensure that everyone involved in conducting research has the knowledge and skills needed to meet high standards of safety, ethics, and accuracy. The second goal is to comply with federal mandates for instruction on the protection of human participants in biomedical and behavioral research and the responsible conduct of research-mandates that initially developed in response to misbehavior, fraud, and scandal.1 There are several different stakeholder groups that require research ethics education.

\section{Community Research Partners}

The label "community research partners" can be applied to organizations as well as individuals, as both have responsibilities for the protection of research participants and the integrity of the research process. For federally funded research that involves human

\footnotetext{
${ }^{1}$ In the 1980s, Congressional hearings on prominent biomedical researchers' reported fraud led Congress to seek to protect U.S. taxpayers' money through increased federal oversight. In 1989, NIH created an Office of Scientific Integrity, and the Department of Health and Human Services (HHS) created its Office of Scientific Integrity Review to investigate allegations of misconduct. In 1982, these two offices were merged into the HHS Office of Research Integrity (ORI), which was given responsibility for "the oversight of research misconduct inquiries and investigations, education and training in the responsible conduct of research, activities designed to promote research integrity and prevent misconduct, and research and evaluation programs" (http://www.ori.dhhs.gov/about/ ORI_Mission.shtml). At the end of the 1990s, the failure of IRBs at several major academic research institutions to provide adequate review and maintain appropriate records led to a temporary shutdown of federally funded research at these institutions. In 1999, the NIH Office for the Protection from Research Risks (OPRR) was reorganized and renamed the Office of Human Research Protections (OHRP) and became part of the Office of the Assistant Secretary for Health (OASH) at HHS. One of OHRP's first efforts to improve the quality of research protections was to require research institutions to provide education to IRB members and researchers, focusing on the protection of research participants (http://www.hhs.gov/ohrp/education/).
} 
participants, education is mandated for anyone "responsible for the design and conduct of the study" (NOT-OD-00-039, Required Education in the Protection of Human Research Participants). Many academic institutions extend this requirement to any individual who will directly interact with research participants or data, regardless of the project's funding source. Institutions fulfill this mandate in a variety of ways and are not required to use any particular curriculum; for example, they may require an existing "packaged" program 2 or may choose to develop their own program to meet the federal requirement. Federal policy has implications not only for individuals who will be involved in the day-to-day activities of research but also for organizational leaders (who may not be as involved).3

The specific research roles of community partners may span the full range of ethical responsibilities, including identifying and recruiting eligible participants; obtaining informed consent; maintaining privacy and confidentiality; collecting, tracking, and collating data; and reporting adverse events. Community partners may also contribute to the development of research questions, study design and methods, and study materials. All of these critically impact study safety and integrity. Although community partners may have significant clinical, service, or other educational experience, they may have little or no prior research experience and will therefore require ethics education early on in a partnership.

Unfortunately, education is too often considered to be a compliance requirement that must be fulfilled before the real work of research can start—and therefore limited to knowledge of the federal regulations. We disagree with this narrow approach to research ethics education. A successful CEnR partnership involves a process of "co-learning" in which both community members and researchers are perceived as experts (Fisher, 1999, 2005). Community partners have specific knowledge of community strengths and vulnerabilities. They possess unique perspectives from which to critique the scientific and social value of a proposed study and inform investigators about the value orientations that guide their reactions to planned procedures (Fisher \& Goodman, 2009; Fisher \& Ragsdale, 2006). If well educated about the ethical challenges that arise in research and the best practices to address them, community partners can serve as a robust link in the chain of protection of research participants and research integrity. Compliance with regulations and certification to conduct research are important goals. However, the primary goals of research ethics

\footnotetext{
2OHRP's policy requires institutions to certify that their IRB members and approved investigators have received appropriate education in the protection of human research participants, ethical practice standards, and federal policies. As this mandate coincided with the increased interest in online educational programs, NIH developed an online training module for its intramural researchers soon after the policy was announced. Many universities accepted completion of that NIH module for their own researchers' certification. Around that same time, a national group of IRB directors and ethics educators envisioned a more comprehensive, adaptable, and "trackable" system of educational modules that universities could modify to meet their own needs. In March 2000, Dr. Paul Braunschweiger at the University of Miami and Karen Hansen at the Fred Hutchinson Cancer Center in Seattle founded the Collaborative IRB Training Initiative (CITI, now "Institutional" instead of "IRB") (https://www.citiprogram.org/aboutus.asp? language $=$ english). Ten content experts joined the collaboration, which developed twelve modules on the protection of human research subjects in biomedical research. In December 2000, the CITI course was made available to research institutions by subscription, effectively creating a national standard for research ethics training. As of November 2011, over 1,100 institutions worldwide rely on the CITI program for training and certification in the protection of human subjects in research in multiple languages, and additional modules are available in research integrity/responsible conduct of research, good clinical practices (GCP), privacy and HIPAA, and the humane care and use of animals in research.

${ }^{3}$ From a regulatory standpoint, an institution (for example, a community-based social service organization or a community-based health clinic) is considered to be "engaged" in research when the involvement of their employees or representatives includes: (1) receipt of federal funding in the form of a grant, contract, or cooperative agreement; (2) "direct intervention for research purposes" with participants that involves invasive or noninvasive procedures or manipulation of the environment; or (3) interaction with participants through "protocol dictated communication" (i.e., recruitment, informed consent, data collection) (Guidance on Engagement of Institutions in Human Subjects Research, http://www.hhs.gov/ohrp/policy/engage08.html). Before becoming engaged in federally supported research that is subject to the Common Rule (Title 45 Part 46, Code of Federal Regulations, Protection of Human Subjects), an institution must hold (or obtain) a Federalwide Assurance (FWA) from the Office of Human Research Protections (OHRP) of the Department for Health and Human Services (HHS). The institution must certify that the research has been reviewed, approved, and will undergo continuing review by an IRB (this can be the IRB of another institution or an independent IRB if an organization does not have its own IRB). In establishing an FWA, an institution assumes responsibility for promoting the rights and welfare of research participants and agrees to comply with all applicable laws, regulations, and policies as well as ethical guidelines for research.
} 
instruction for CEnR partners should be to promote knowledge and understanding of good research practices and ultimately create—as theologian Paul Ramsey advocated in his seminal work on research ethics (1970)—-true "co-adventurers" in the research enterprise.

\section{Academic Investigators}

Most university-based investigators have likely completed research ethics educational requirements prior to getting involved in a CEnR project. Some institutions may also require continuing human research protections education or refresher courses, and many professionals seek out additional training relevant to specific methods, populations, and topics. However, research ethics courses may not address ethical issues and standards of practice that are unique to $\mathrm{CEnR}$, and CEnR education may not relate ethical issues that arise in practice to federal research regulations. Academic investigators are often not aware of the specific ethical challenges that community research partners face in the field, nor do they necessarily have ready solutions. Academic investigators require specific ethics education relevant to the CEnR context.

\section{Community-Academic Research Partnerships}

Educating community partners in isolation-for example, through web-based educational modules - limits opportunities for dialogue, questions, and the direct application of concepts to the research project on which they will be working. Any disconnect or separation between ethics education and protocol-specific training may send a subtle message to community partners that ethics education is merely a requirement to be met. Ideally, ethics education should be delivered to academic-community research partnerships, and the comprehensive educational needs of all partners should be discussed at the onset of the partnership. Ethics instruction should be role-specific but should also situate individuals' roles in context within the overall project team.

\section{Institutional Review Boards}

IRBs are comprised of individuals with a variety of disciplinary backgrounds. This means that many IRB staff and members are not familiar with the particularities of CEnR. This lack of familiarity creates challenges for investigators in presenting their work to IRBs as well as deficits in IRB review of CEnR projects (Flicker et al., 2007; Guta et al., 2010; Khanlou \& Peter, 2005; Malone et al., 2006; Shore, 2007; Wolf, 2010). For example, a misunderstanding of CEnR may lead an IRB to harbor misgivings about the competencies of community partners and therefore require "more" rather than "more relevant" training (Dolor, Smith, \& Neale, 2008; Yawn et al., 2009). This can create administrative burdens for community partners and exacerbate mistrust among all parties (Solomon \& Piechowski, 2011). IRBs that are unfamiliar with CEnR may be limited in their ability to assist CEnR teams in tackling ethical problems that emerge during study design (e.g., deciding whether or not to include a control community) or project implementation (e.g., handling potential breaches of confidentiality). Proposed changes to the federal regulations for human research (Department of Health and Human Services, 2011) may provide more flexibility for behavioral and social science research, and community-academic partnerships will be looking to local IRBs to help them understand how the regulations may affect their research.

Open dialogue between community-academic partnerships and IRBs can increase the appropriateness and rigor of ethical review of CEnR (Wolf, 2010). University-based investigators should educate IRB members regarding the unique aspects of their work and negotiate alternative training strategies for their community partners (Dolor, Smith, \& Neale, 2008). IRBs should also be involved in efforts to educate community partners about research, especially institutional policies and procedural requirements. The extensive amount of external oversight that is characteristic of research may be unfamiliar to 
community partners, and bringing IRBs and community partners together can put a human face on what is often an enigmatic review process (Hyatt et al., 2009).

\section{How Should Research Ethics Education Be Delivered? \\ There are significant gaps in knowledge regarding best practices for research ethics instruction}

Although NIH requires education for individuals in certain research roles, NIH does not endorse any particular instructional programs. Institutions are considered to be in the best position to determine what programs are appropriate for their needs. NIH does not delineate specific content that should be included in education (NOT-OD-00-039, Required Education in the Protection of Human Research Participants). The evidence base for what constitutes effective instruction in the protection of research participants and scientific integrity is weak (Antes et al., 2009; Antes et al., 2010; Heitman et al., 2007). Instructional programs currently utilized by academic institutions have not been well evaluated; no "gold-standard" has been identified, although certain programs are considerably more popular than others (Braunschweiger \& Goodman, 2007). In education on the responsible conduct of research, 4 there is significant variability in topics covered (Epstein, 2008; Grossman et al., 2004) and no unified approach to instruction (Sunderland et al., 2011).

\section{One size does not fit all}

The most urgent need may be for educational programs tailored to the unique needs of community partners. Due to the variety of roles, communities, research topics, designs, and partnership structures, an effective curriculum must be flexible and responsive to the ethical challenges of local contexts and learning needs. At the same time, IRBs have been more apt to recognize consistent, one-size-fits-all training programs. While some may criticize these "packaged" educational products, they are effective at providing a low-cost, lowcommitment, and highly efficient way to streamline and track the delivery of education to a large number of individuals, hence their popularity with academic institutions. The widespread use of the online Collaborative Institutional Training Initiative (CITI) Program has created the impression for many institutions that CITI is the required training program. In their efforts to cover the core elements of research protections, educators may overlook the fact that NIH policy gives individual institutions responsibility and authority for identifying what needs to be taught and how best to provide instruction but does not prescribe a particular curriculum.

Community research partners are obviously different from academic partners in some important ways, but little work has been done to identify their specific research ethics educational needs. Several academic institutions, government bodies, and research teams have created research ethics educational materials specifically for community research

\footnotetext{
${ }^{4}$ Following a policy update issued in November 2009, NIH now requires that all trainees, fellows, participants, and scholars receiving support through an NIH training, career development award (individual or institutional), research education grant, or dissertation research grant receive substantive instruction (defined as eight or more hours-in person/face-to-face and not online) in the "responsible conduct of research" (RCR) (http://grants.nih.gov/grants/guide/notice-files/NOT-OD-10-019.html). NIH policy defines RCR as "the practice of scientific investigation with integrity," which "involves awareness and application of established professional norms and ethical principles in the performance of activities related to scientific research." (Required RCR instruction covers broad nine topic areas, including conflict of interest; policies regarding human subjects, live vertebrate animal subjects, and safe laboratory practices; mentor/trainee responsibilities and relationships; collaborative research including collaborations with industry; peer review; the acquisition, management, sharing, and ownership of research data; research misconduct (fabrication, falsification, and plagiarism) and policies for handling misconduct; responsible authorship and publication; and the scientist as a responsible member of society, contemporary ethical issues in biomedical research, and the environmental and societal impacts of scientific research. These new topics areas reorganized and expanded upon earlier recommendations to emphasize policy in human and animal research and laboratory safety; the social role of biomedical scientists, ethical issues in biomedicine, and the social and environmental impact of research (http://grants1.nih.gov/grants/guide/notice-files/NOT-OD-10-019.html).
} 
partners, but these are primarily project, population, or institution specific and therefore must be adapted for use with other types of partners (see Table 2). We are not aware of any curriculum that has been thoroughly evaluated or is endorsed by recognized research ethics experts that is widely accepted by IRBs as a substitute for the standard human subjects protection training required of all key research personnel. While institution-based and community-specific trainings may continue to proliferate and be shared, a national consensus on the basic minimal requirements for content, preferred formats and modes of delivery, and standards for assessment of research ethics education for community partners is needed to provide guidance and consistency, as well as facilitate recognition and acceptance of alternative trainings by IRBs.

There is often a disconnect between training offered by an institution in fulfillment of the requirements and the information and skills that are actually needed to work on a CEnR project. For example, preliminary research has shown that CEnR studies may have more data accuracy and completeness problems than those that use traditional academic-based research assistants (Brugge et al., 2010), but standard research ethics education rarely covers data integrity issues in any detail as research personnel working in academic settings learn these norms and skills through other means. Common sense also suggests that community partners must have basic "research literacy" before they can truly contribute to research-or benefit from instruction on more advanced or specific topics like ethical principles or informed consent (Goodman, Dias, \& Stafford, 2010; Ross et al., 2010c). Much of the research ethics instruction offered by academic institutions may not be perceived by community partners as relevant or useful; this assertion is based on published reports (Alexander \& Richman, 2008) as well as our own experience and that of colleagues.

In many settings, modifying curricula initially designed for academic researchers or graduate students is not always a viable or appropriate solution (Merritt et al., 2010). Current standardized programs contain much information that is not directly relevant to CEnR studies (especially those that do not involve medical intervention), and many do not include practical information that people involved in the day-to-day work of community-based studies need to know in order to do their jobs well (True, Alexander, \& Richman, 2011). For example, research ethics education may deliver the message that protecting participant confidentiality is important but not necessarily explain how to accomplish this when working in the community—literally on the streets and out of cars—and interacting with research participants who are part of their regular social networks (Edwards, Lund, \& Gibson, 2008; Terpstra et al., 2011). There is often a disconnect between how recruitment, informed consent, and data collection tools are developed for institutional review board (IRB) submission and how they are implemented in the field. Written research protocols tend to focus on the language in the consent form but omit details of the recruitment and informed consent process that are essential for those who will actually be doing that work. Community research partners are not always provided with adequate guidance regarding what to do once they get in the field, the specific challenges they may face, or tools for resolving dilemmas (Fisher et al., 2012; True, Alexander, \& Fisher, 2012)—for example, how to handle if a potential participant slams a door in their face, insists that they want to participate but will not take the time to read the consent form (or have it read to them), or appears to lie to meet inclusion criteria.

\section{Formal instruction is not enough to ensure compliance with rules}

Mentoring and leadership from experienced community and academic investigators as well as institutional policies and climate can also influence the ethics and integrity of research (Clinical and Translational Science Awards Community Engagement Key Function Committee Task Force on the Principles of Community Engagement, 2011). However, even 
less is known about how to change institutional climate than how to provide optimal research ethics education (Geller et al., 2010).

The following recommendations for best practices and a research agenda result from our extensive review of the theoretical and empirical literatures related to research ethics education and ethical issues in CEnR. Through the group writing process, we have identified certain areas where evidence is sufficient to support concrete recommendations. We have also identified significant gaps in knowledge, suggested which gaps deserve priority, and offered specific research strategies for filling those gaps.

\section{Best Practices}

\section{Ethics Education Across Stakeholder Groups}

We have argued that community research partners, academic investigators, and IRB members need research ethics education that is CEnR-focused and tailored to their learning needs. However, while the specific instructional needs and learning styles of these stakeholder groups may be different, we argue that their instruction should not occur in isolation from each other. Opportunities for information exchange are crucial and should be encouraged. Time should be taken to engage in co-learning regarding the people, institutions, and communities involved in the partnership in order to foster a culture of scientific integrity and transparency.

\section{Specific Recommendations for Educating Community Research Partners}

Every community is unique, and each research protocol is different, but ultimately unifying principles, unified approaches, and core tools are needed. Therefore, any curricular materials or resources developed specifically for community partners should emphasize consistency with traditional standardized programs in terms of learning objectives and topics, but they should also be flexible enough to allow customization to community and project contexts and learning needs. Research ethics education should be tailored to partners' unique roles, responsibilities, backgrounds, and learning styles. While research is needed to identify optimal instructional methods and content, we can make some preliminary recommendations in these areas (see Table 3).

First, to decrease burden, education should be focused on the specific research context. Content should be relevant to immediate roles and responsibilities, research design and setting, and participation population. Second, education for community partners with no or limited research experience should be as protocol-specific as possible. Individuals who are new to research cannot be expected to extrapolate broad ethical principles or relate examples from other research settings to their specific situations. Program materials should anticipate those ethical dilemmas that may reasonably occur during fieldwork, and instructors should teach to these specific issues. Key process issues related to scientific integrity, such as what constitutes falsification/fabrication of data, accurate completion and handling of data forms, and tracking participant data and incentive payments, should be covered. Third, research ethics education should include hands-on opportunities to practice new skills (e.g., approaching a potential participant and obtaining informed consent) and receive concrete feedback. In order to minimize burden, instruction regarding such operational issues could be provided in the form of on-the-job training by a variety of experts, including research subject advocates (RSAs),5 experienced clinical research coordinators, or communityengagement specialists located centrally within academic institutions. Fourth, community research partners involved in day-to-day fieldwork activities should have ongoing opportunities for discussion among themselves and with study investigators to debrief. Ideally, this dialogue will create a feedback loop about ethical issues that arise in practice and strategies for addressing them. These discussions should also include approaches to 
managing feelings of moral distress (and stress more generally). Fifth, ethical issues that are unique to CEnR should be integrated into educational programs for all stakeholder groups. Before this can be accomplished, more research is needed to determine the most salient problems, characterize the dilemmas, and identify potential ethical solutions that are consistent with the values of both academic researchers and community partners. Lastly, research ethics education should focus on communication; an important goal of education for community partners should be empowerment to ask questions and speak up when they see potential ethical pitfalls.

\section{Research Agenda}

Research ethics education should be evidence-based both in terms of the topics covered and instructional methods employed. To this end, we propose the following research agenda and emphasize that studies in all of these areas could greatly benefit from a partnership approach (see Table 4).

First, there is a critical need for large-scale evaluations of existing, quality research ethics educational programs for all stakeholders but community partners in particular. Experimental methods should be used to identify which instructional methods (e.g., online programs versus face-to-face training; individual role versus team-based approaches) are most effective for each group.

Second, more information is needed regarding community partners' various research roles in order to more appropriately tailor education to specific levels of engagement.

Third, further exploration is needed to determine how university-based investigators, community partners, and IRB members may differently define, approach, and resolve ethical issues. Some fundamental differences in professional culture, expertise, and the interpretation of research risks and benefits may influence ethical analysis. This groundwork can identify areas for educational focus, particularly in terms of communication, and support development of tools such as models for facilitating consensus when partners fundamentally disagree about the translation or prioritization of basic ethical principles.

Fourth, further investigation of prospective participant views of research is needed. Such work may uncover research practices that are scientifically sound but inconsistent with community values. Such practices may create participation barriers due to community concerns regarding threats to autonomy, group stigmatization, or participant distress. These concerns may not be readily discerned through professional logic or scientific inference. Such research will require investigator openness to community knowledge and opinion that may challenge traditional scientific procedures and perspectives and require novel and unconventional yet rigorous methodologies (Fisher, 1999, 2005; Fisher et al., in press; Goldberg-Freeman et al., 2007).

\footnotetext{
${ }^{5}$ Since the creation of the Research Subject Advocate (RSA) role by NIH in 2000, RSAs have filled a variety of needs in human research protection programs at academic centers, ranging from direct advocacy work with participants, to auditing, improving the transparency of research, and educating researchers in human research protections (National Center for Research Resources Division for Clinical Research Resources, 2005; Neill, 2003; O’Lonergan, 2003). Within CTSA-funded institutions, Research Subject Advocacy has increasingly been organized around best practice functions that include the integration of human research protection policies and educational activities across institutional entities and acting as a resource to research participants and investigators (CTSA Regulatory Workgroup, 2008). Some RSA programs also train community research partners regarding the protection of research participants. Other RSA programs, particularly those that are too large to engage participants individually, use their RSA resources to host broad outreach programs designed to raise the visibility of research, engage communities in setting priorities, educate partners and communities about the potential benefits and risks of research, and explain the protections and procedures in place to protect research participants (Winkler, 2011).
} 
Fifth, some specific challenges to the protection of participants and the integrity of the research process that arise in the field in CEnR projects require systematic investigation. Currently, published case studies rather than rigorous research are the primary source of information regarding unique ethical issues in CEnR. National surveys of CEnR projects could identify common problems and describe differences among various research designs, populations, and settings. Qualitative research with stakeholders could identify areas in need of ethical clarification. Below we outline four broad areas of exploration that could greatly contribute to the development of content for CEnR ethics education (numbered as 5.1-5.4).

5.1 Information regarding the nature of financial relationships among academic institutions/investigators, research projects, community-based agencies, and individual community partners should be collected. Details regarding contractual agreements, employment status, salary, and benefits as well as arrangements for sharing power, resources, and decision making among stakeholders are crucial to understanding the full picture of CEnR ethics (Alexander \& Richman, 2008).

5.2 More research is needed to determine how dual roles (i.e., community partners' research and nonresearch roles) or competing priorities can create conflicts of interest that pose harm to research participants and/or the integrity of research data. The potential effect of recruitment through community-based organizations on participants' perceptions of voluntariness, the understanding of key elements of informed consent (e.g., right to withdraw, benefits), and breaches of confidentiality must be examined (Anderson, 2010). Research is needed on research participants' perceptions of "insider" (e.g., community partners) versus "outsider" (e.g., graduate research assistants) recruiters and the effects of these perceptions on participant enrollment and retention, quality of informed consent, voluntariness, trust in research, compliance with study requirements, data quality, and perceived value of research (Alexander \& Richman, 2008; Simon \& Mosavel, 2010). This information can inform best practices for the training, selection, and compensation of individuals who recruit research participants and obtain informed consent process (Molyneux, Kamuya, \& Marsh, 2010; Simon \& Mosavel, 2010).

5.3 A better understanding of actual harms to individuals qua community membership as well as harms to communities is needed. Research can identify strategies for mediating these risks (Fantuzzo, McWayne, \& Childs, 2006; Fisher et al., in press; Mohatt \& Thomas, 2006) as well as potential guidelines for considering community-level risks in the IRB review process.

5.4 Research is needed on moral distress. This could explore questions such as how competing responsibilities are balanced, particularly when service providers (or individuals with a service orientation) are involved in recruitment, informed consent, and data collection (Fisher et al., 2012). Strategies for identifying and reducing moral distress could be incorporated into research ethics education.

\section{Educational Implications}

Throughout this manuscript, we have documented how currently available research ethics education programs fail to meet the needs of all groups that have a role in CEnRcommunity research partners, academic investigators, and IRB members. Ideally, curricular materials and activities should reflect the realities and particularities of CEnR; identify potential areas of difference and disagreement among stakeholder groups; recognize and promote the value of protecting research participants, the integrity of the research process, and the quality of data; and embrace CEnR principles of co-equal partnership. Robust, 
tailored educational resources and opportunities can enhance the potential of community partners to be effective agents for the ethical conduct of research and support the selfdetermination of communities and individuals to engage and participate (or not) in research.

\section{Acknowledgments}

The project described was supported by the National Center for Research Resources and the National Center for Advancing Translational Sciences, National Institutes of Health, through grants to multiple institutions. The content is solely the responsibility of the authors and does not necessarily represent the official views of the NIH. The manuscript was approved by the CTSA Consortium Publications Committee.

The authors thank the co-sponsors of the third annual 2011 Community-Engaged Research Workshop, "Communication in Community-Engaged Research"- the Journal of Empirical Research on Human Research Ethics, the Hobby Center for Public Policy at University of Houston, Public Responsibility in Medicine and Research (PRIM\&R), and Community-Campus Partnerships for Health-for allowing several of us to present our ideas at the conference. We also thank conference attendees for their responsiveness and enthusiasm.

\section{Biographies}

Alice Ammerman is Professor in the Department of Nutrition and Director of the Center for Health Promotion and Disease Prevention at the University of North Carolina at Chapel Hill, which is a Prevention Research Center. She is also a faculty member in the Community Academic Resources for Engaged Scholarship (CARES) group of the North Carolina Translational and Clinical Sciences.

Emily E. Anderson is Assistant Professor at the Neiswanger Institute for Bioethics and Health Policy, Loyola University Chicago, Stritch School of Medicine. Before completing a $\mathrm{PhD}$ in health care ethics at Saint Louis University, she worked with several communityengaged research projects in Chicago, IL. Her research interests include empirical research in bioethics, IRB policy and processes, health disparities, and ethical issues in public health research and practice, and she has served on several IRBs.

James M. DuBois is the Hubert Maeder Professor of Health Care Ethics in the Albert Gnaegi Center for Health Care Ethics and Director of the Bander Center for Medical Business Ethics. He also directs the Center for Clinical Research Ethics within the Institute for Clinical and Translational Science at Washington University in St. Louis. His research interests include mental health research ethics, assessing outcomes of ethics education, and understanding and preventing wrongdoing in research.

Celia B. Fisher is Marie Ward Doty University Chair, Professor of Psychology and founding Director of the Fordham University Center for Ethics Education. Her research programs focus on ethical issues and the well-being of vulnerable populations including ethnic minority youth and families, active drug users, medically ill youth, college students at risk for drinking problems, and adults with impaired consent capacity.

Elizabeth Heitman is Associate Professor and Director of Clinical and Research Ethics in the Center for Biomedical Ethics and Society at Vanderbilt University Medical Center. A longtime research ethics educator, she is Chair Elect of the National CTSA Collaborative's Clinical Research Ethics Key Function Committee and a member of its Education Materials Subcommittee.

Bonnie Jones is a Research Assistant for the Community Academic Resources for Engaged Scholarship (CARES) group of the North Carolina Translational and Clinical Sciences (NC TraCS) Institute and the Center for Health Promotion and Disease Prevention at the University of North Carolina at Chapel Hill. 
Rhonda G. Kost is the Clinical Research Officer for the Rockefeller University Center for Clinical and Translational Science. She co-chairs the Action Committee for Community Engaged Research and helped to foster community-based research with a practice-based research network. Her research interests include human subjects protection training effectiveness, and the development-validated, participant-centered measures of the quality of the research participant experience.

Mary Ellen Lawless is part of the Community Engagement core of the NIH-sponsored Clinical Translational and Science Collaborative Award (CTSC) at Case Western Reserve University. Her work includes providing CREC education for community researchers. She has assisted with developing materials specifically tailored to community researcher's project study roles. She has experience in clinical research with diverse individuals and a special interest in the delivery of culturally appropriate health care and research interventions.

Cornelia Ramsey is Assistant Professor and the Community Research Liaison in Virginia Commonwealth University's Center for Clinical and Translational Research (CCTR). She has 20 years of experience working in communities and with community-based organizations in Virginia, North Carolina, and South Carolina to address health promotion, disease prevention, and health inequities within vulnerable, underserved populations. A qualitative researcher, she is also works with African American communities and several American Indian tribal communities in central Virginia to explore past experiences and perceptions of research and how that history continues to impact the health and well-being of those communities today.

Lainie Friedman Ross is the Carolyn \& Matthew Bucksbaum Professor of Clinical Ethics, Professor, Departments of Pediatrics, Medicine, Surgery, and the College; Associate Director, MacLean Center for Clinical Medical Ethics, University of Chicago. She currently serves as the co-director of the University of Chicago Clinical and Translational Science Award (CTSA). She conceived of this project with Emily Anderson in her role as cochairperson of the Ethics and Community Engagement Workgroup, a subcommittee of the Clinical Research Ethics Workgroup with co-reporting responsibilities to the Community Engagement Workgroup, two workgroups of the national CTSA consortium. Her research interests focus on research ethics, pediatric ethics, transplantation ethics, and ethical issues in genetics.

Stephanie Solomon is Assistant Professor at the Albert Gnaegi Center for Health Care Research Ethics at Saint Louis University and Adjunct Professor of Medicine at Washington University School of Medicine. Her areas of interest include community-based participatory research, especially in the areas of biobanking, human subjects regulations, and the role of the public in scientific and medical decision making, as well as the appropriate uses of public deliberation and biobanking consent processes.

\section{References}

Alexander L, Richman K. Ethical dilemmas in evaluations using indigenous research workers. American Journal of Evaluation. 2008; 29(1):73-85.

Anderson E. The role of community-based organizations in the recruitment of human subjects: Ethical considerations. American Journal of Bioethics. 2010; 10(3):20-21. [PubMed: 20229409]

Antes A, Murphy S, Waples E, Mumford M, Brown R, Connelly S, Devenport L. A meta-analysis of ethics instruction effectiveness in the sciences. Ethics and Behavior. 2009; 19(5):379-402. [PubMed: 19838311] 
Antes A, Wang X, Mumford M, Brown R, Connelly S, Devenport L. Evaluating the effects that existing instruction on responsible conduct of research has had on ethical decision making. Academic Medicine. 2010; 85(3):519-526. [PubMed: 20182131]

Bean S, Silva D. Betwixt and between: Peer recruiter proximity in community-based research. American Journal of Bioethics. 2010; 10(3):18-19. [PubMed: 20229408]

Braunschweiger P, Goodman K. The CITI program: An international online resource for education in human subjects protection and the responsible conduct of research. Academic Medicine. 2007; 82(9):861-864. [PubMed: 17726392]

Brugge D, Cole A. A case study of community-based participatory research ethics: The Healthy Public Housing Initiative. Science and Engineering Ethics. 2003; 9(4):485-501. [PubMed: 14652901]

Brugge D, Kapunan P, Babcock-Dunning L, Matloff R, Cagua-Koo D, Okoroh E, et al. Developing methods to compare low-education community-based and university-based survey teams. Health Promotion Practice. 2010; 11(5):645-653. [PubMed: 19129433]

Buchanan D, Miller F, Wallerstein N. Ethical issues in community-based participatory research: Balancing rigorous research with community participation in community intervention studies. Progress in Community Health Partnerships: Research, Education, and Action. 1997; 1(2):153-160.

Clinical and Translational Science Awards Community Engagement Key Function Committee Task Force on the Principles of Community Engagement. Principles of community engagement. 2nd ed.. Atlanta: Centers for Disease Control and Prevention; Agency for Toxic Substances and Disease Registry; 2011.

CTSA Regulatory Workgroup. Meeting summary, January 25, 2008. 2008. Available at http:// www.ctsaweb.org/uploadedfiles/CTSARegWG_20080125_final.pdf.

Department of Health and Human Services, Office of the Secretary, Food and Drug Administration. Human subjects research protections: Enhancing protections for research subjects and reducing burden, delay, and ambiguity for investigators. 2011. Available at http://www.gpo.gov/fdsys/pkg/ FR-2011-07-26/html/2011-18792.htm.

Dolor RJ, Smith PC, Neale AV. Institutional review board training for community practices: Advice from the Agency for Health Care Research and Quality practice-based research network listserv. Journal of the American Board of Family Medicine. 2008; 21(4):345-352. [PubMed: 18612061]

Downie J, Cottrell B. Community-based research ethics review: Reflections on experience and recommendations for action. Health Law Review. 2001; 10(1):8-17. [PubMed: 15739305]

DuBois J, Bailey-Burch B, Bustillos D, Campbell J, Cottler L, Fisher C, et al. Ethical issues in mental health research: The case for community engagement. Current Opinion in Psychiatry. 2011; 24:208-214. [PubMed: 21460643]

Edwards K, Lund C, Gibson N. Ethical validity: Expecting the unexpected in community-based research. Pimatisiwin: A Journal of Aboriginal and Indigenous Community Health. 2008; 6(3):1730 .

Epstein S. The rise of "recruitmentology": Clinical research, racial knowledge, and the politics of inclusion and difference. Social Studies of Science. 2008; 38(5):801-832. [PubMed: 19227822]

Fantuzzo, J.; McWayne, C.; Childs, S. Scientist-community collaborations: A dynamic tension between rights and responsibilities. In: Trimble, J.; Fisher, C., editors. The handbook of ethical research with ethnocultural populations and communities. Thousand Oaks, CA: Sage; 2006. p. 27-50.

Fisher, C. Decoding the ethics code: A practical guide for psychologists. 2nd ed.. Thousand Oaks, CA: Sage; 2012.

Fisher, C. Participant consultation: Ethical insights into parental permission and confidentiality procedures for policy relevant research with youth. In: Lerner, R.; Jacobs, F.; Wertlieb, D., editors. Applied developmental science: An advanced textbook. Thousand Oaks, CA: Sage; 2005. p. 113-138.

Fisher, C. Relational ethics and research with vulnerable populations. In: National Bioethics Advisory Commission. , editor. Research involving persons with mental disorders that may affect decisionmaking capacity, vol. II. Rockville, MD: 1999. p. 29-49.

Fisher C, Busch N, Brown J, Jopp D. Applied developmental science, social justice, and socio-political well-being. Applied Developmental Science. 2012; 16(1):54-64. 
Fisher, C.; Goodman, S. Goodness-of-fit ethics for non-intervention research involving dangerous and illegal behaviors. In: Buchanan, D.; Fisher, C.; Gable, L., editors. Research wtih high-risk populations: Balancing science, ethics, and law. Washington, DC: American Psychiatric Association; 2009. p. 25-46.

Fisher, C.; Ragsdale, K. A goodness-of-fit ethics for multicultural research. In: Trimble, J.; Fisher, C., editors. The handbook of ethical research with ethnocultural populations and communities. Thousand Oaks, CA: Sage; 2006. p. 3-26.

Fisher, C.; True, G.; Alexander, L.; Fried, A. Moral distress and the responsible conduct of community-based drug use research. Paper presented at the Quest for Research Excellence; March 15-16; Washington, DC. 2012.

Fisher C, Wallace S. Through the community looking glass: Re-evaluating the ethical and policy implications of research on adolescent risk and psychopathology. Ethics \& Behavior. 2000; 10(2): 99-118. [PubMed: 11841105]

Flicker S, Travers R, Guta A, McDonald S, Meagher A. Ethical dilemmas in community-based participatory research: Recommendations for institutional review boards. Journal of Urban Health. 2007; 84(4):478-493. [PubMed: 17436114]

Geller G, Boyce A, Ford DE, Sugarman J. Beyond "compliance": The role of institutional culture in promoting research integrity. Academic Medicine. 2010; 85(8):1296-1302. [PubMed: 20671455]

Gikonyo C, Bejon P, Marsh V, Molyneux S. Taking social relationships seriously: Lessons learned from the informed consent practices of a vaccine trial on the Kenyan coast. Social Science and Medicine. 2008; 67(5):708-720. [PubMed: 18362046]

Goldberg-Freeman C, Kass N, Tracey P, Ross G, Bates-Hopkins B, Purnell L, et al. "You've got to understand community": Community perceptions on "breaking the disconnect" between researchers and communities. Progress in Community Health Partnerships: Research, Education, and Action. 2007; 1(3):231-240.

Goodman M, Dias J, Stafford J. Increasing research literacy in minority communities: CARES Fellows Training Program. Journal of Empirical Research on Human Research Ethics. 2010; 5(4):33-40. [PubMed: 21133785]

Grossman D, Agarwal I, Biggs V, Brenneman G. Ethical considerations in research with socially identifiable populations. Pediatrics. 2004; 113(1):148-151. [PubMed: 14702468]

Guta A, Wilson M, Flicker S, Travers R, Mason C, Wenyeve G, O'Campo P. Are we asking the right questions? A review of Canadian REB practices in relation to community-based participatory research. Journal of Empirical Research on Human Research Ethics. 2010; 5(2):35-46. [PubMed: 20569148]

Heitman, E.; McKieran, L. Module 4: Community-based practice and research: Collaboration and sharing power. In: Jennings, B.; Kahn, J.; Mastroianni, A.; Parker, L., editors. Ethics and public health: Model curriculum. Washington, DC: Association of Schools of Public Health; 2004.

Heitman E, Olsen C, Anestidou L, Bulger R. New graduate students' baseline knowledge of the responsible conduct of research. Academic Medicine. 2007; 82(9):838-845. [PubMed: 17726387]

Hood N, Brewer T, Jackson R, Wewers M. Survey of community engagement in NIH-funded research. Clinical and Translational Science. 2010; 3(1):19-22. [PubMed: 20443949]

Hyatt R, Gute D, Pirie A, Page H, Vasquez I, Dalembert F. Transferring knowledge about human subjects protections and the role of institutional review boards in a community-based participatory research project. American Journal of Public Health. 2009; 99(Suppl. 3):S526-S531. [PubMed: 19890152]

Khanlou N, Peter E. Participatory action research: Considerations for ethical review. Social Science and Medicine. 2005; 60(10):2333-2340. [PubMed: 15748680]

Lakes K, Vaughan E, Jones M, Burke W, Baker D, Swanson J. Diverse perceptions of the informed consent process: Implications for the recruitment and participation of diverse communities in the national children's study. American Journal of Community Psychology. 2012; 49(1-2):215-232. [PubMed: 21671109]

Landy D, Sharp R. Examining the potential for exploitation by local intermediaries. American Journal of Bioethics. 2010; 10(3):12-13. [PubMed: 20229405] 
Levine R. Clinical trials and physicians as double agents. Yale Journal of Biological Medicine. 1992; 65(2):65-74.

Lindau S, Makelarski J, Chin M, Desautels S, Johnson D, Johnson W, et al. Building communityengaged health research and discovery infrastructure on the South Side of Chicago: Science in service to community priorities. Preventive Medicine. 2011; 52(3-4):200-207. [PubMed: 21236295]

Malone R, Yerger V, McGruder C, Froelicher E. "It's like Tuskegee in reverse": A case study of ethical tensions in institutional review board review of community-based participatory research. American Journal of Public Health. 2006; 96(11):1914-1919. [PubMed: 17018816]

Merritt M, Labrique A, Katz J, Rashid M, West K, Pettit J. A field training guide for human subjects research ethics. PLoS Medicine. 2010; 7(10):1-4.

Minkler M. Ethical challenges for the "outside" researcher in community-based participatory research. Health Education and Research. 2004; 31(6):684-697.

Mohatt, G.; Thomas, L. "I wonder, why would you do it that way?" Ethical dilemmas in doing participatory research with Alaska Native communities. In: Trimble, J.; Fisher, C., editors. The handbook of ethical research with ethnocultural populations and communities. Thousand Oaks, CA: Sage; 2006. p. 93-116.

Molyneux S, Kamuya D, Marsh V. Community members employed on research projects face crucial, often under-recognized, ethical dilemmas. American Journal of Bioethics. 2010; 10(3):24-25. [PubMed: 20229411]

National Center for Research Resources, Division for Clinical Research Resources. Research Subject Advocates: Guidelines for the General Clinical Research Centers Program. Bethesda, MD: 2005.

Neill K. Research Subject Advocate: A new protector of research participants. Accountability in Research. 2003; 10(3):159-174. [PubMed: 14979318]

O'Lonergan T. Creative solutions: Research Subject Advocates: Increase in reports of human subject protection deficiencies bring scrutiny as well as more efforts at education and support. Protecting Human Subjects. 2003; 3(8):10-11.

Phillips T. Protecting the subject: PDR and the potential for compromised consent. American Journal of Bioethics. 2010; 10(3):14-15. [PubMed: 20229406]

Quinn S. Ethics in public health research: Protecting human subjects: The role of community advisory boards. American Journal of Public Health. 2004; 94(6):918-922. [PubMed: 15249289]

Ramsey, P. The patient as person. New Haven, CT: Yale University Press; 1970.

Ross L, Loup A, Nelson R, Botkin J, Kost R, Smith G, Gehlert S. The challenges of collaboration for academic and community partners in a research partnership: Points to consider. Journal of Empirical Research on Human Research Ethics. 2010a; 5(1):19-31. [PubMed: 20235861]

Ross L, Loup A, Nelson R, Botkin J, Kost R, Smith G, Gehlert S. Human subjects protections in community-engaged research: A research ethics framework. Journal of Empirical Research on Human Research Ethics. 2010b; 5(1):5-17. [PubMed: 20235860]

Ross L, Loup A, Nelson R, Botkin J, Kost R, Smith G, Gehlert S. Nine key functions for a human subjects protection program for community-engaged research: Points to consider. Journal of Empirical Research on Human Research Ethics. 2010c; 5(1):33-47. [PubMed: 20235862]

Shore N. Community-based participatory research and the ethics review process. Journal of Empirical Research on Human Research Ethics. 2007; 2(1):31-41. [PubMed: 19385890]

Shore N. Re-conceptualizing the Belmont Report: A community-based participatory research perspective. Journal of Community Practice. 2006; 14(4):5-26.

Silka L, Renault-Caragianes P. Community-university research partnerships: Devising a model for ethical engagement. Journal of Higher Education Outreach and Engagement. 2006; 11(2):171183.

Simon C, Mosavel M. Community members as recruiters of human subjects: Ethical considerations. American Journal of Bioethics. 2010; 10(3):3-11. [PubMed: 20229402]

Solomon S, Piechowski P. Developing community partner training: Regulations and relationships. Journal of Empirical Research on Human Research Ethics. 2011; 6(2):23-30. [PubMed: 21680974] 
Strauss R, Sengupta S, Quinn S, Geoppinger J, Spaulding C, Kegeles S, Millett G. The role of community advisory boards: Involving communities in the informed consent process. American Journal of Public Health. 2001; 91(12):1938-1943. [PubMed: 11726369]

Sunderland N, Catalano T, Kendall E, McAuliffe D, Chenoweth L. Exploring the concept of moral distress with community-based researchers: An Australian study. Journal of Social Service Research. 2011; 37(1):73-85.

Terpstra J, Coleman K, Simon G, Nebeker C. The role of community health workers (CHWs) in health promotion research: Ethical challenges and practical solutions. Health Promotion Practice. 2011; 12(1):86-93. [PubMed: 19346410]

True, G.; Alexander, L.; Fisher, C. Addressing ethical challenges in urban drug addiction research: Lessons from community research workers. Paper presented at the Quest for Research Excellence; March 15-16; Washington, DC. 2012.

True G, Alexander L, Richman K. Misbehaviors of front-line research personnel and the integrity of community-based research. Journal of Empirical Research on Human Research Ethics. 2011; 6(2): 3-12. [PubMed: 21680972]

Ulrich C, Hamric A, Grady C. Moral distress: A growing problem in the health professions? Hastings Center Report. 2010; 40(1):20-22. [PubMed: 20166512]

Weijer C. Protecting communities in research: Philosophical and pragmatic challenges. Cambridge Quarterly of Health Care Ethics. 1999; 8(4):501-513. [PubMed: 10513308]

Winkler, S. "Making sausages from silos." Presentation at CTSA Regulatory Knowledge Key Function meeting, January 12. 2011. Available at http://www.ctsaweb.org/uploadedfiles/ 05_04_1015AM_Sabune_Harvard\%20Catalyst\%20Reg\%20KFC\%20F2F\%20RSA.pdf.

Wolf L. The research ethics committee is not the enemy: Oversight of community-based participatory research. Journal of Empirical Research on Human Research Ethics. 2010; 5(4):77-86. [PubMed: 21133789]

Yawn B, Graham D, Bertram S, Kurland M, Dietrich A, Wollan P, et al. Practice-based research network studies and institutional review boards: Two new issues. Journal of the American Board of Family Medicine. 2009; 22(4):453-460. [PubMed: 19587261] 


\section{TABLE 1}

Authors' Related Roles and Experience.

\begin{tabular}{ll}
\hline Current or Past Experience & Authors \\
\hline Member or chair of IRB & Anderson, Solomon, Heitman, DuBois, Kost, Ramsey, Ross \\
Research ethics consultation service & Anderson, Solomon, Heitman, DuBois, Kost, Ammerman, Ross \\
Principal Investigator on CEnR project & Fisher, Kost, Ramsey, Ammerman, Ross \\
CEnR a primary area of research & Fisher, Kost, Lawless, Ramsey, Ammerman (CEnR ethics: Anderson, Solomon) \\
Member of Community Advisory Board & Anderson, Solomon, Lawless, Ramsey, Jones, Ammerman \\
Member of advisory board for CEnR project & DuBois, Fisher, Kost, Lawless, Jones, Ammerman \\
Involved in development of research ethics curriculum & Anderson, Solomon, Heitman, DuBois, Fisher, Kost, Lawless, Ramsey, Jones \\
\hline
\end{tabular}


TABLE 2

CEnR Ethics Education (in alphabetical order).

\begin{tabular}{ll}
\hline Curriculum & Reference/Web Address (Organization) \\
\hline CIRTification: & http://go.uic.edu/CIRTification \\
Community Involvement & (University of Illinois Chicago in partnership with Northwestern University, \\
Human Research & University of Chicago, Rush University, \& Loyola University of Chicago) \\
Protetions
\end{tabular}

Description

- Uses train-the-trainer model and includes participant workbook

- Intended for the spectrum of CEnR partners/roles

- Developed by one of this paper's authors (EA)

Ethical Protections in Community-Engaged Research
Solomon, S., \& Piechowski, P. (2011). Developing community partner training: Regulations and relationships. Journal of Empirical Research on Human Research Ethics, 6(2), 23-30.

(University of Michigan)
To be used for group instruction

- Developed by one of this paper's authors (SS)

\begin{abstract}
Ethics and Research in the http://ori.hhs.gov/education/products/mass_cphs/training_staff/index.htm Community

Alexander, L., \& Richman, K. (2008). Ethical dilemmas in evaluations using indigenous research workers. American Journal of Evaluation, 29(1), 73-85.

(Office of Research Integrity, Bryn Mawr College, Massachusetts College of Pharmacy and Health Sciences)
\end{abstract}

- Completed online

- Available in Spanish

- Intended for research workers in community-based trials

$\begin{array}{ll}\text { Faith Moves Mountains } & \begin{array}{l}\text { Hatcher, J., \& Schoenberg, N. (2007). Human subjects protection training } \\ \text { for community workers: An example from "Faith Moves Mountains." } \\ \text { Progress in Community Health Partnerships, 1(3), 257-265. }\end{array} \\ & \text { (University of Kentucky) }\end{array}$

Field Training Guide

Merritt, M., Labrique, A., Katz, J., Rashid, M., West, K., \& Pettit, J. (2010) A field training guide for human subjects research ethics. PLoS Medicine, 7(10), 1-4.

http://www.jhsph.edu/sebin/u/p/Field\%20Guide_25Feb10.pdf

(Johns Hopkins University)
- $\quad$ Brief written guide

- Intended for field workers/data collectors in community-based trials

\section{Keeping Research on Track: A Guide for Aboriginal \& Torres Strait Islander Peoples about Health Research Ethics}

http://www.nhmrc.gov.au/publications/synopses/e65syn.htm

(Australian Health and Medical Research Council)
- $\quad$ Intended for Aboriginal and Torres Strait Islander peoples
Project TRES: Training in http://www-rohan.sdsu.edu/ gra/grad/research/projecttresinfo.html Research Ethics \& Standards
Terpstra, J., Coleman, K., Simon, G., \& Nebeker, C. (2011). The role of community health workers (CHWs) in health promotion research: Ethical challenges \& practical solutions. Health Promotion Practice, 12(1), 86-93.

(San Diego State University)
- $\quad$ Available in webbased or hard copy format for use as either a stand-alone, directed self-study training or in conjunction with group training

- nitially developed for community health 


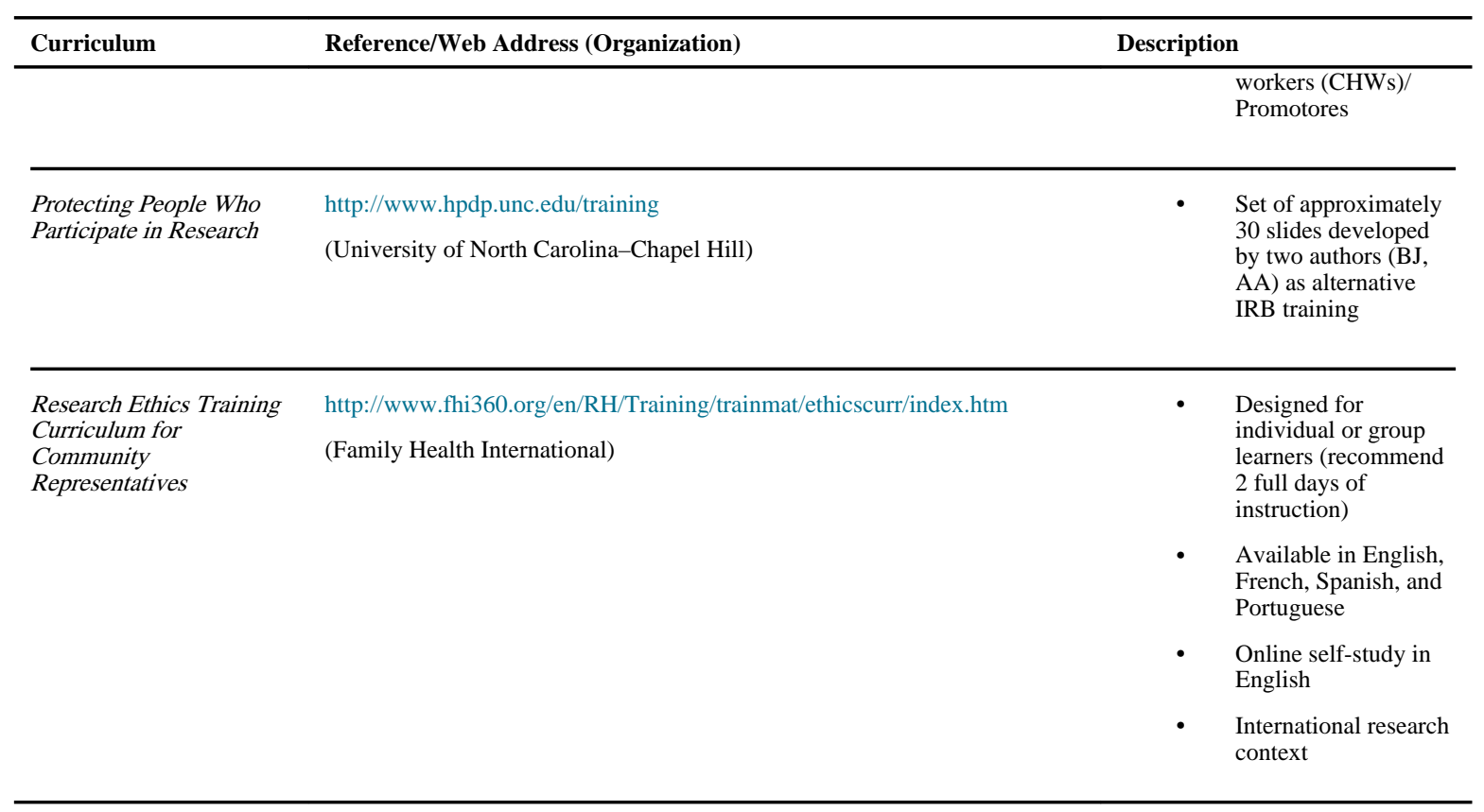


TABLE 3

Best Practices for Research Ethics Education for Community-Engaged Research.

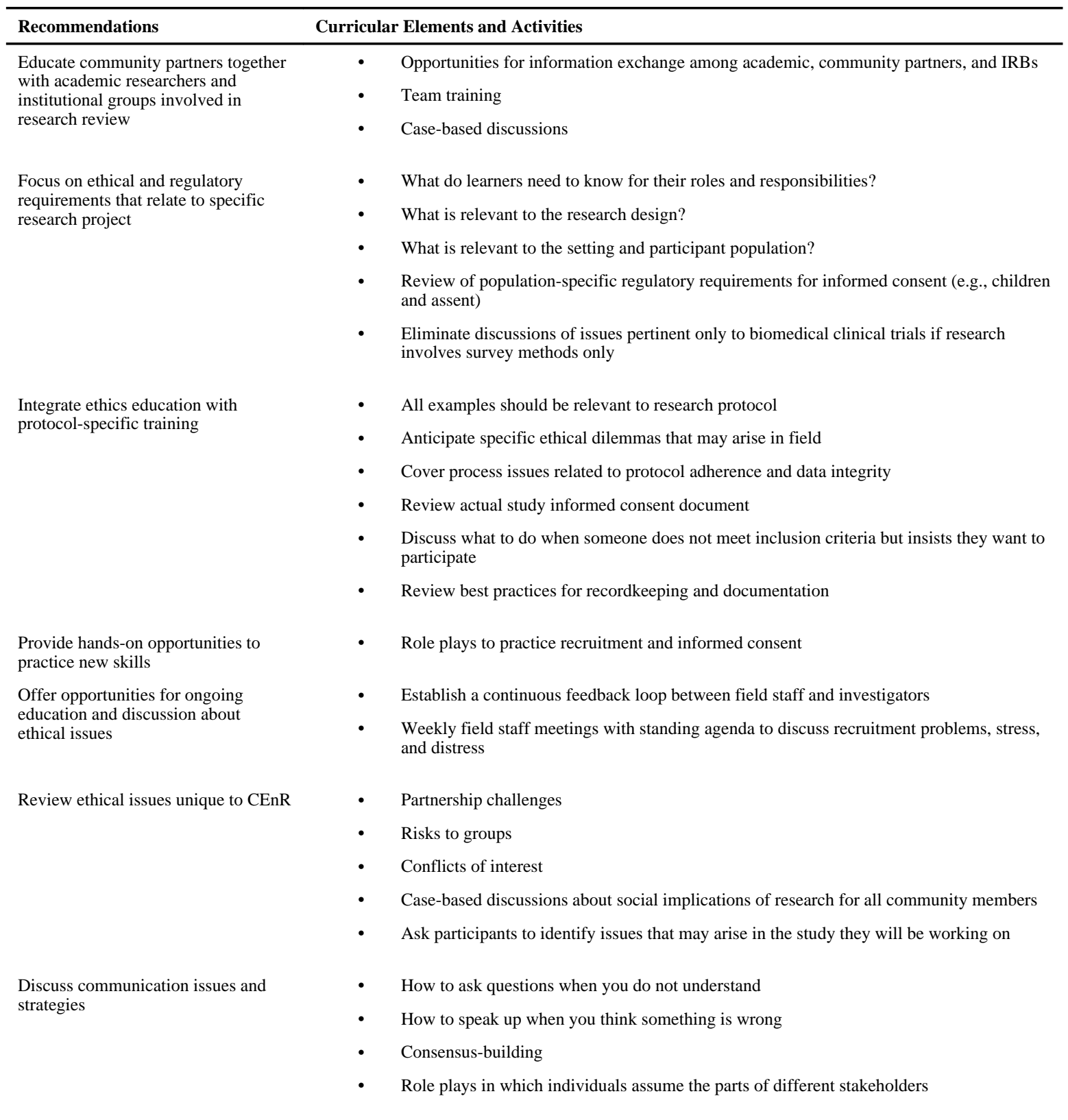


TABLE 4

Research Agenda for Ethical Issues in CEnR.

Research on Educational Methods

- Evaluate research ethics education programs

- Identify effective instructional methods for different learner groups

- Gather information on research roles played by community partners in order to tailor education to specific levels of engagement

Research on Defining and Resolving Ethical Issues

- $\quad$ Explore how different stakeholders define, approach, and resolve ethical issues

- Test and refine models for facilitating consensus when stakeholders disagree

Research on Participant Views

- Determine actual and potential participants' opinions, preferences, and values regarding (for example)

- $\quad$ autonomy and informed consent

- $\quad$ privacy and confidentiality protections

- $\quad$ barriers to research participation

- $\quad$ group stigmatization resulting from research participation or findings

- $\quad$ potential for research to cause distress

\section{Research on Ethical Challenges Unique to CEnR}

- Describe the types of financial relationships that exist between academic-community partners

- Examine the potential harms of community partners' dual roles and competing priorities

- Describe real and potential cases of harm to communities and individuals qua community membership

- Identify sources of potential stress and distress among community research partners and develop strategies for minimizing stress and distress

- Characterize common problems and describe differences among research designs, populations, and settings

- Recognize and explore areas in need of ethical clarification 